\title{
Holistic approach towards micro- and macroscopic properties of the cerebrospinal fluid
}

\section{Herbowski L*}

Neurosurgery Department District Hospital, Arkonska 4, 71-455 Szczecin, Poland

The actual behavior and parameters of the cerebrospinal fluid contained in the subarachnoid cavity and in the ventricles of the brain are evaluated from different physicochemical perspectives. The temperature, volume and pressure of the cerebrospinal fluid lie in the field of macroscopic studies, whereas its molecular electrostatics, Brownian motion, diffusion and cohesion are the domain of the microworld. While both physical states might seem unrelated, ultimately the micro-scale phenomena underlie the macroscopic ones. If it weren't for movements of all molecules suspended in the cerebrospinal fluid, there would be no intracranial pressure exerted on intracranial structures. Even though the macrostatic parameters are general and simple to examine while microstatic ones complicated and difficult to describe, both of these physical states are inseparably connected.

For over a dozen years together with my colleagues-physicists and on my own I have undertaken clinical and laboratory studies to assess these two physicochemical states. Macroscopic examinations have been based on the analysis of intracranial pressure in patients with suspicion of normotensive hydrocephalus in connection with environmental conditions (atmospheric pressure, air temperature) and Earth's geomagnetic field. Additionally, I have analyzed if and to what extent different body thermal points of the patient have a potential impact on the circulation of the cerebrospinal fluid. The microscopic evaluation was based on the identification of macromolecules and their movement in the cerebrospinal fluid and the recognition of eclectrostatic phenomena. The macro and micro phenomena are, in turn, a reflection of events on the nano scale, where the atoms are in constant motion. This molecular movement of atoms is called "heat movement". Thus, what plays an important role are the electrostatic forces and surface tension. Phenomena occurring here, on a nanoscopic scale, are based on quantum mechanics. The first scientist who observed this movement of suspended particles in 1872 was Robert Brown, after whom the motion is called "Brownian motion" and the moving particles are conventionally called Brown particles. Marian Smoluchowski described these movements in his pioneering and deserving of the Noble Prize work as "shaking" movements [1]. Indeed, elementary particles are moving chaotically in a zig-zag fashion, forming components of the cerebrospinal fluid and colliding with each other (Figure 1).

The frequency of collisions between the particles is estimated at an average of one thousand times per second. The view under a light microscope depicts organic macromolecules called biomolecules, both proteins and cells contained in the cerebrospinal fluid and their fragments and conglomerates.

The observed biomolecules range in size from several hundred nanometers (larger protein particles) to a few micrometres (cells). The observation was conducted by means of Nikon Eclipse 600 microscope,

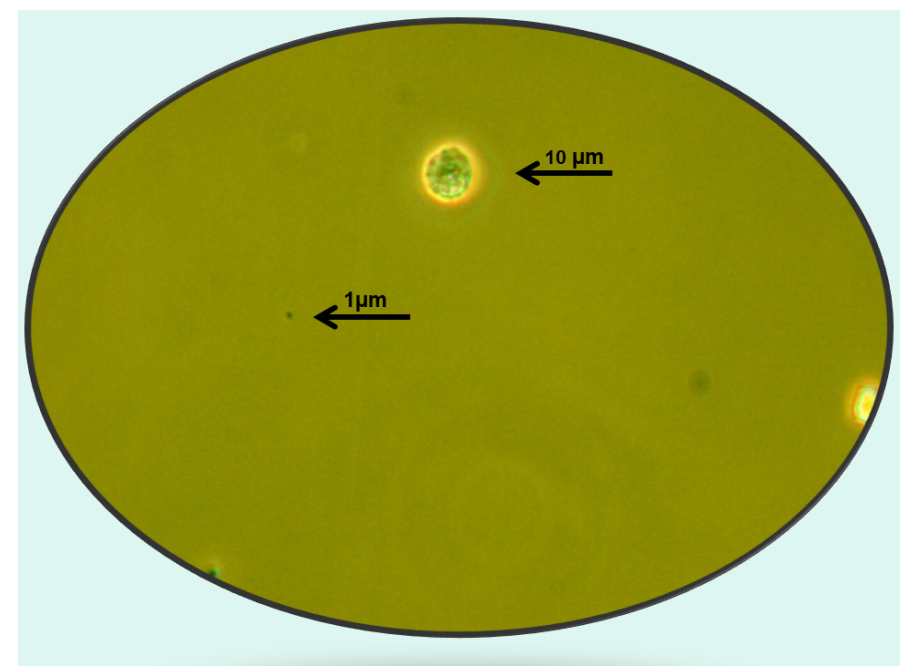

Figure 1. Macromolecules are visible through the light microscope: a large lymphoid cell and a small protein [Own material]

coupled through a digital camera to a computer equipped with the Lucia v. 4.6 software from Laboratory Imaging s.r.o. - the equipment of the Institute of Marine Physics and the Environment, the Institute of Physics of the University of Szczecin. For the purpose of computer image analysis a few drops of the cerebrospinal fluid were placed in the Bürker chamber by the use of micropipette. Observing the field of view during several dozens of seconds, it was possible to capture the movement of biomolecules in the plane of the fluid layer. These are the Brownian motions, both slower movements of larger objects like cells (Figures 2 and 3) and faster movements of small objects like proteins (Figure 4).

The phenomenon worth highlighting is the diversity of the objects suspended in the cerebrospinal fluid. The static parameters of the molecules contained in the cerebrospinal fluid collected from 27 neurosurgical patients with disorders of the cerebrospinal fluid circulation (after prior consent of the bioethics commission for the study) were analyzed under the microscope. It turned out that in 1 $\mathrm{mm}^{3}$ there are on average 134 (from 35 to 740 ) objects with an average surface area of $25.9 \mu \mathrm{m}^{2}$. All static parameters of molecules as the

*Correspondence to: Leszek Herbowski M.D., Ph.D, Neurosurgery Department District Hospital, Arkonska 4, 71-455 Szczecin, Poland, E-mail: leszekherbowski@data.pl

Received: March 01, 2019; Accepted: March 18, 2019; Published: March 21, 2019 


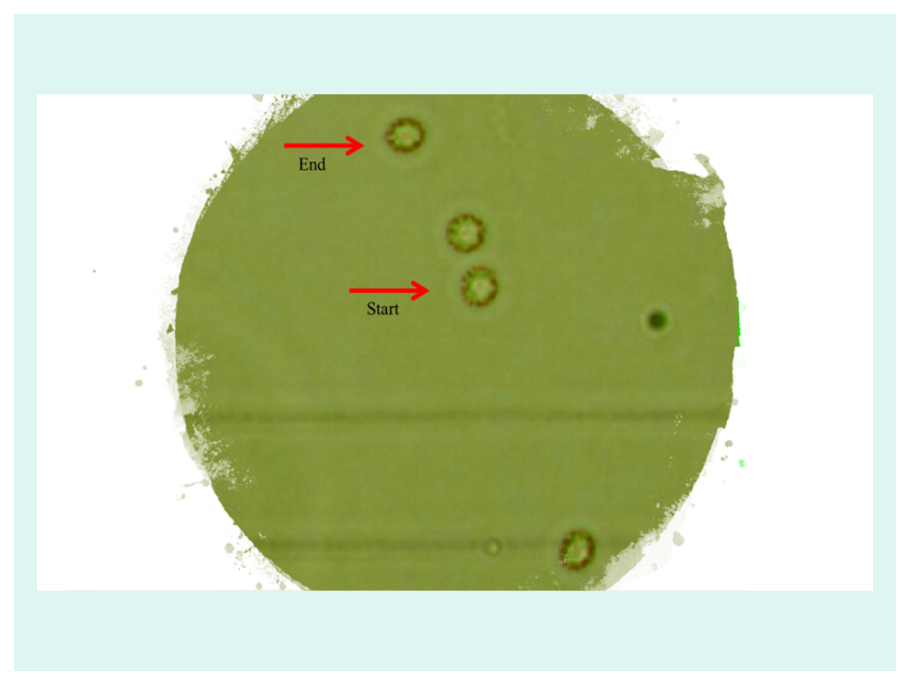

Figure 2. The image from the light microscope with the application of three photos from the starting position (START) to the final position (END) with the intermediate position. The cell with a diameter of $7 \mu \mathrm{m}$ suspended in the cerebrospinal fluid moves about twenty micrometres (mean shift vector $0.6 \mu \mathrm{m}$ ) [Own material]

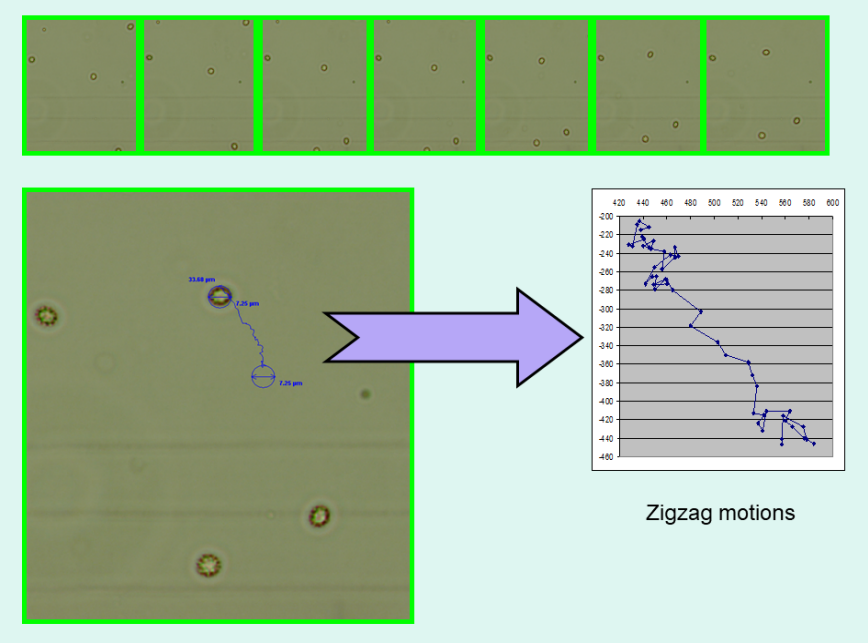

Figure 3. The next phases of the Macromolecule's movement (Brownian motion) with the designation of subsequent zig-zags [Own material]

average number of objects, average density of objects, average total and relative total area of objects, average total length of perimeter of objects, mean relative total length of objects perimeter were published in Proceedings of Spie in 2007 [2].

In 1904, Smoluchowski was the first to draw attention to and describe the underlying fluctuating molecular motion, which pioneered the development of stochastic physics. On the other hand, molecular particles colliding with and surrounding each other form a macroscopic state, which in turn is based on the laws of classical Newton's mechanics. The observed behavior of molecules at the "microworld" level is random or unpredictable, which remains in contrast to the description of the "macroworld", where the phenomena are completely deterministic, predictable. Such simultaneous coexistence of both interpenetrating worlds - microstate and macrostate - is still an incomprehensible and undiscovered contemporary mystery.

The first paper to describe observed in vivo Brownian motions of macromolecules, i.e. cells and protein particles suspended in the cerebrospinal fluid was published in 2009 [3]. The suspended macromolecules, called hard ones according to Ohshima's theory of electrophoresis, are surrounded by solvent ions. The main goal of this work was to describe this ionic cloud forming a double layer of Stern which surrounds the biomolecule that matches the arachnoid. The thickness of the Stern layer estimated for macromolecules directly attached to the arachnoid is several micrometres. The observed changes in the Stern layer thickness indicate a continuous fluctuation in the density of electric charges on both sides of the layer.

In another paper focused on molecular electrical phenomena [4] there was a precise description of the double layer of Stern around the biomolecule suspended in the cerebrospinal fluid (Figure 5).

As shown in the paper the Stern layer around hard biomolecules is formed by positive sodium ions and therefore the thickness of the Stern layer is estimated at 0.366 nanometer. In this case, the outer layer around the Stern layer, the so-called Gouy layer, is formed by negative chloride ions. These molecular electrochemical phenomena described in both publications are pioneering contribution to the study of the cerebrospinal fluid at the microscopic level.

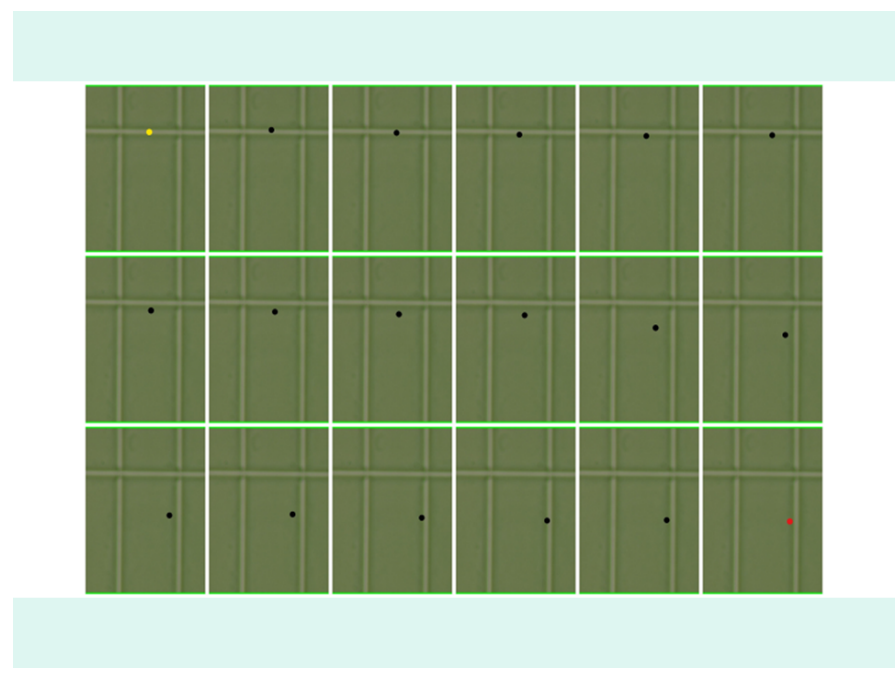

Figure 4. Microscopic eighteen photos of the Brownian motion from the starting position (yellow dot) to the final one (red dot) with intermediate positions (black dots). Protein with a diameter of only $1 \mu \mathrm{m}$ suspended in the cerebrospinal fluid moves by several dozen micrometres (mean $0.9 \mu \mathrm{m}$ of displacement vector) [Own material]

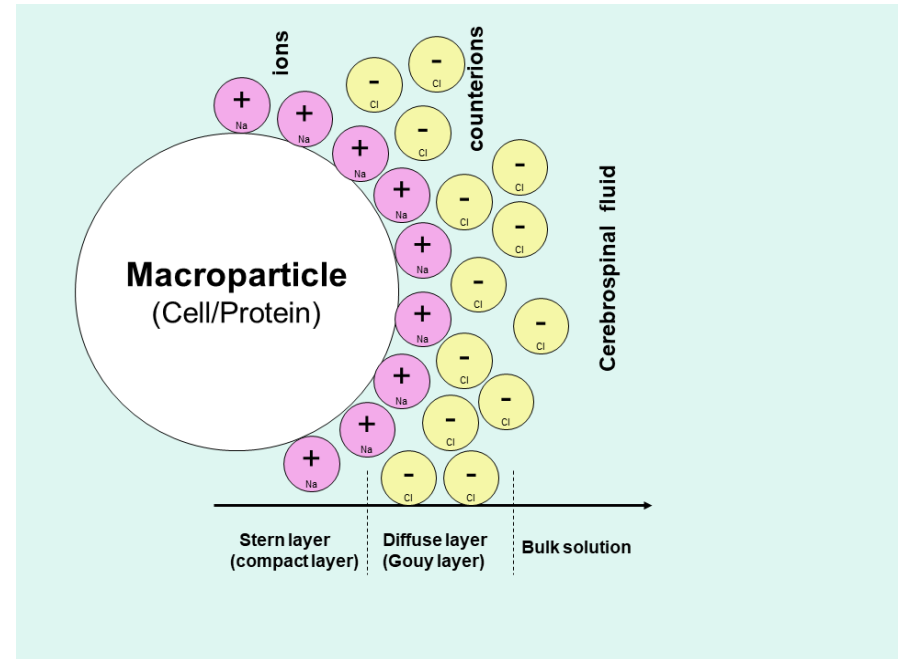

Figure 5. Schematic representation of Stern's double electrical layer around biomolecules suspended in the cerebrospinal fluid [Own artwork] 
In the next paper [5] the main focus was on analyzing images of the cerebrospinal fluid in two groups. The first group consisted of patients $(\mathrm{N}=6)$ with enlargement of the ventricular system and the second group of patients $(\mathrm{N}=13)$ with internal hydrocephalus. It turned out that there were significant differences between the two groups in the range of distribution curve of the suspended biomolecules diameter. Namely, in the case of the ventricular system expansion, particles with a diameter of 5-10 $\mu \mathrm{m}$ prevailed, and in the hydrocephalus group, smaller particles with a diameter of $0.5-5 \mu \mathrm{m}$ dominated. This finding led to a conclusion that the distribution curve of molecules can be a helpful and fast tool allowing to differentiate clinically expressed hydrocephalus from the radiological widening of the ventricular system.

However, linking my clinical practice with the biophysical and physicochemical basis of molecular phenomena in the cerebrospinal fluid was reflected in a manuscript already in 2005 [6]. Not only was the physical property of the cerebrospinal fluid (its viscosity) described, but there was also analyzed the influence of the shape of the molecule on its viscosity and fluidity. A retrospective analysis of 13 neurosurgical patients with post-traumatic bleeding was possible after obtaining the consent of the bioethical commission. It was found out that after elevation the patient's body to $30^{\circ}$ and $50^{\circ}$ angles, increases in the cerebrospinal fluid pressure measured from the lumbar puncture were significantly higher in the group with minor bleeding than in patients with massive bleeding. This, in turn, triggered a discussion on how elementary phenomena at the molecular level, such as fluid viscosity, diffusion of suspended molecules in the fluid, and mutual adhesion between molecules and surrounding brain membranes, affect changes in macroscopically measured the cerebrospinal fluid pressure from the lumbar puncture. In result, it was stated that the admixture of the blood in the cerebrospinal fluid (Figure 6) reduces the reserve of compensation mechanisms of the intracranial-intraspine system.

The mechanism consists in increasing internal resistance (coherence forces) in pathological cerebrospinal fluid and increasing the adhesion between fluid and surrounding membranes. The admixture of blood in the cerebrospinal fluid contributes to the increase of its viscosity, which is determined by Einstein's formula. Following the subarachnoid hemorrhage, the viscosity of such a fluid can be increased by at least two and a half times. In turn, Fick's diffusion law shows that the admixture of blood in the cerebrospinal fluid reduces its fluidity. The description of molecular phenomena served to explain the changes in the measured cerebrospinal fluid pressure observed in clinical practice. This was the first in the world attempt to link intracranial and intraspine microstate with a fluid macrostate. None of the current cerebrospinal fluid researchers has analyzed in such a way the presented biological mechanisms from the molecular perspective.

In parallel to studies regarding the cerebrospinal fluid in micro scale, studies of its macrostate were carried out [7]. The aim of the study was to examine the influence of atmospheric pressure and ambient temperature (T-ambient) on the parameters of intracranial volume - pressure homeostasis, including intracranial pressure, both mean (ICPmean), systolic (ICPsyst) and diastolic (ICPdiast) pressure, mean arterial pressure (BPmean), systolic BPsyst) and diastolic (BPdiast) blood pressure, and body temperature (T-patient). Figure 7 presents curves of individual parameters in 14 neurosurgical patients with suspected normal pressure hydrocephalus.

Until that publication the influence of atmospheric pressure on intracranial parameters had not been studied or analyzed. The pioneering research in this area revealed that in the range of 746-768

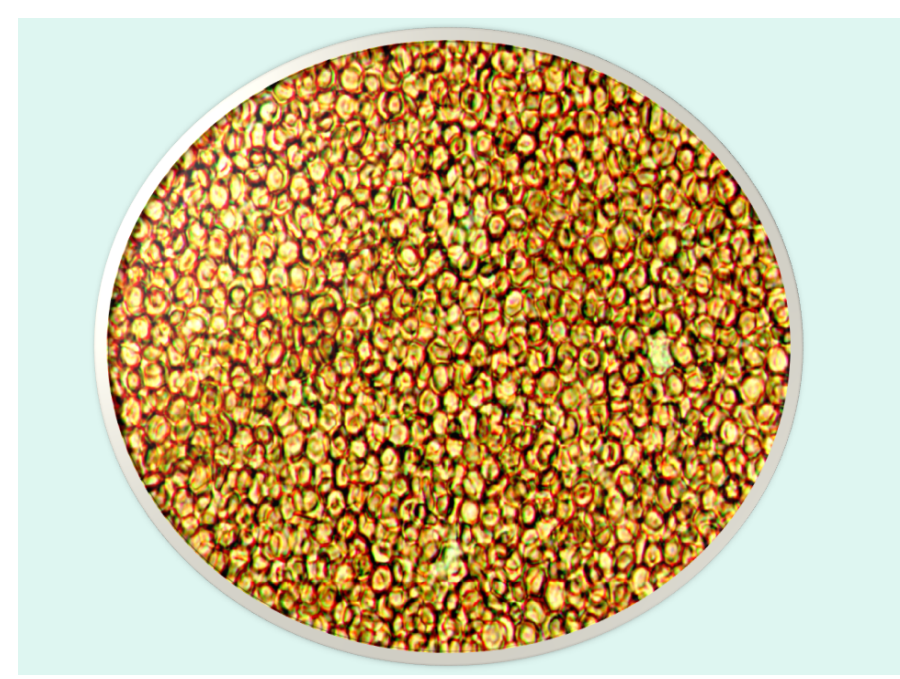

Figure 6. The entire field of view under the light microscope is covered by morphing elements of the blood, i.e. red blood cells (massive subarachnoid hemorrhage) [Own material]

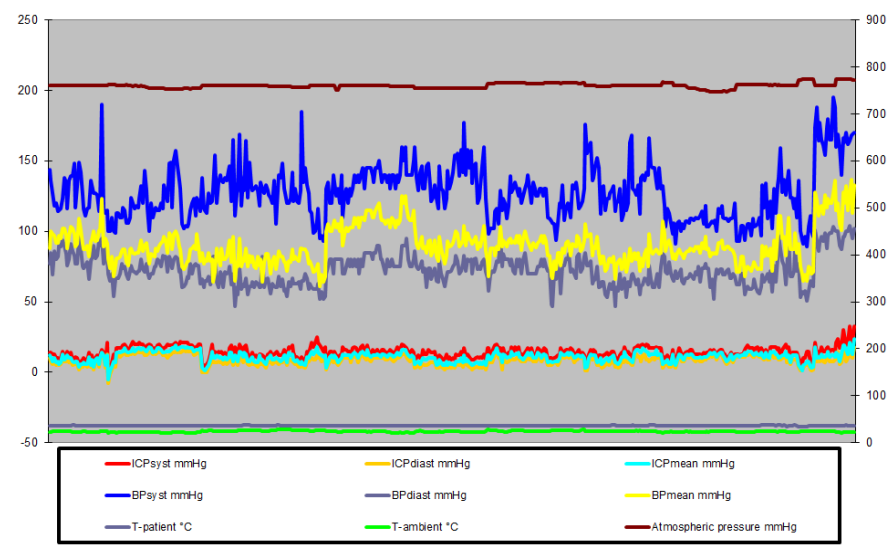

Figure 7. The measured variables (492 observations). The average atmospheric pressure was $759.8 \mathrm{mmHg}$. Mean intra-spinal pressure (mean ICP) was $11.05 \mathrm{mmHg}$, systolic ICP $14.49 \mathrm{mmHg}$, diastolic ICP $8.45 \mathrm{mmHg}$. Mean BP was $90.05 \mathrm{mmHg}$, systolic BP 127.45 $\mathrm{mmHg}$, diastolic BP $72.48 \mathrm{mmHg}$. The average body temperature was $36.56^{\circ} \mathrm{C}$ and the average ambient temperature was $24.1^{\circ} \mathrm{C}$

$\mathrm{mmHg}$ the atmospheric pressure significantly influences the behavior of intracranial and arterial pressure. Firstly, at atmospheric pressure below $755 \mathrm{mmHg}$, the increase in atmospheric pressure correlates significantly with the reduction of intracranial pressure and increase in arterial pressure. Secondly, at atmospheric pressure between 756 $\mathrm{mmHg}$ and $766 \mathrm{mmHg}$, the increase in atmospheric pressure correlates significantly with lowering blood pressure and increasing intracranial pressure. Thirdly, at an atmospheric pressure of $766 \mathrm{mmHg}-768$ $\mathrm{mmHg}$, the increase in atmospheric pressure significantly correlates with a reduction in intracranial pressure and an increase in blood pressure. Last but not least, at atmospheric pressure of $770 \mathrm{mmHg}-774$ $\mathrm{mmHg}$, intracranial homeostasis parameters as well as arterial pressure remain insensitive to external conditions. Importantly, a link between atmospheric pressure and blood flow was also observed. Atmospheric pressure below $770 \mathrm{mmHg}$ significantly correlates with blood flow measured by mean brain perfusion pressure. A contrario, at pressures above $770 \mathrm{mmHg}$, the brain's perfusion pressure remains constans.

The relation between absolute intracranial pressure and absolute atmospheric pressure based on a clinical study was presented in another paper [8]. The reference for the measured relative intracranial 
pressure is ambient atmospheric pressure. However, the reference for the measured absolute atmospheric pressure is the vacuum where the pressure is equal to $0 \mathrm{mmHg}$. Hence, the reference for absolute intracranial pressure (absolute ICP) is also a vacuum. Thus, the absolute intracranial pressure is equal to the sum of the absolute atmospheric pressure plus the gauge intracranial pressure.

This is the first study taking into account the absolute values of the pressures tested. It turned out that the relationship between atmospheric pressure and intracranial pressure is significantly high (correlation coefficient equal to 0.87 with $\mathrm{p}<0.05$ ) - (Figure 8).

In addition, this study allowed for the first time to positively verify the thesis that changes in absolute intracranial pressure are associated with seasons (spring, summer, autumn, winter). A positive correlation was found between atmospheric and intracranial pressure and each season.

The average values of atmospheric and intracranial pressure were higher in the winter, spring and summer than in the autumn. The highest intracranial and atmospheric pressure was recorded in the summer and the lowest in the autumn. Differences between atmospheric and intracranial pressures were positive in all seasons except for the autumn $(-1.4 \mathrm{hPa}$ difference). The intersection of both curves indicates the limit of abrupt intracranial pressure close to $1007.0 \mathrm{hPa}$ which resembles the so-called reference point for this pressure.

The thermodynamic aspect of convection of the cerebrospinal fluid has not been yet the subject of scientific considerations and studies. In the analysis from 2011 the descending and ascending movements of the cerebrospinal fluid in the spinal canal were described taking into account temperature differences at both ends of neural tube [9]. Skin temperature measurements were carried out in 16 medium-sized volunteers of 38.8 years in the sacral area (Temp.S) at the level of the spinal process of the fifth lumbar vertebra and in the frontal area of the head (Temp G); the examinations were carried out using a wireless thermometer. The average temperature in the sacral region was $34.4^{\circ} \mathrm{C}$, while in the frontal area $36.0^{\circ} \mathrm{C}$. The average temperature difference at both ends of the neuron tube was $1.6^{\circ} \mathrm{C}$. There were statistically significant differences in temperature between the head and sacral ends at the 0.95 confidence level. In this work the thesis on the movement of the cerebrospinal fluid in the spinal canal was presented. The vertebral canal from the thermodynamic point of view can be divided into two parts: the outer one adhering to the skin and the inner one adhering to the internal organs. In the outer (dorsal) part, the temperature decreases from the neck to the sacral part. The temperature drop is $1.6^{\circ} \mathrm{C}$. The cerebrospinal fluid movement is molecular and occurs under the influence of gravity and the Brownian motion. Brownian motion under the influence of decreasing temperature becomes less intense, the speed of fluid molecules becomes smaller, and thus the free path decreases, the number of collisions per unit of time decreases, the distances between molecules decrease. Thus, both the density of fluid and its weight increase. This contributes to the lowering of the cerebrospinal fluid itself. The movement of the cerebrospinal fluid in the anterior (ventral) part of the spinal canal is caused by its heating by internal organs. The fluid temperature in this part of the channel is higher than the temperature in the outer part of the channel at the same level. This means that the fluid density at the same level is lower as a result of the volume increase. The higher in the canal, the higher the fluid temperature becomes, which contributes to the growth of Brownian movements, and in consequence to the fluid density decrease. Brownian motion contributes to moving the fluid up. At the interface of cool liquid molecules with warmer ones, as a result of heat transfer, the liquid cools down gradually. In contrast, warming from the ventral side of the spinal canal, the fluid rises in the cephalic direction and moves in a circular motion.

The studies presented above are examples of interdisciplinary approach linking the clinical practice with physics laws and statistical verification. On the basis of achieved results it was possible to put forward an innovative thesis related to the cerebrospinal fluid's properties. The conclusion goes beyond the insights coming from other researchers' publications so far, at the same time enabling to identify the variables influencing physicochemical features of the fluid. Namely, the structure of the Stern layer on the border of molecules suspended in the cerebrospinal fluid and brain membranes is affected by the surrounding atmospheric pressure and electromagnetic radiation of the Sun (Figure 9).

This conception is a bridge between the microworld and macroworld scientifically indicating the overarching factors not considered in the cerebrospinal fluid's research before.

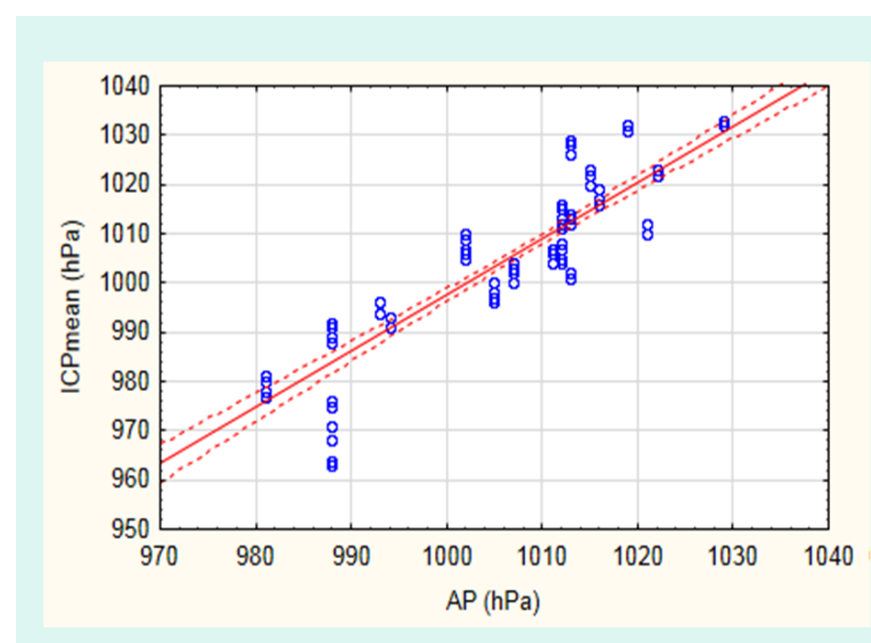

Figure 8. Spearman's correlation curve between intracranial pressure (ICP) and atmospheric pressure (AP). $95 \%$ confidence interval (CI) from 0.82 to 0.90

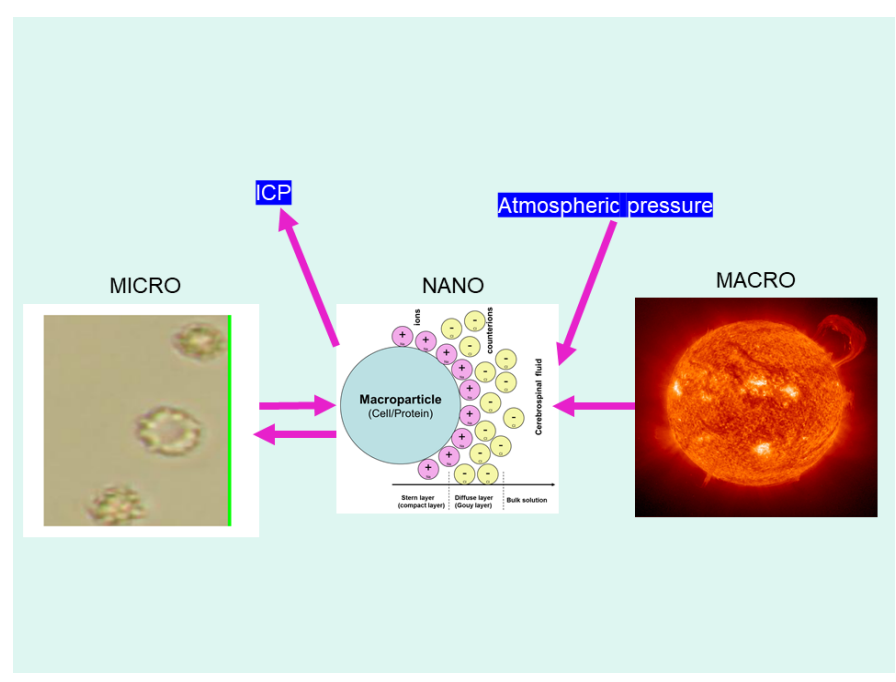

Figure 9. Between the cerebrospinal fluid and molecules suspended in it (MICRO image on the left side of the figure) and the surrounding tissues, a double electric Stern layer is created with a specific fluctuating electric charge density (NANO image in the middle of the figure). The density of this electric charge may be influenced by the external electromagnetic field of the Earth and the Sun (MACRO image on the right side of the figure) 


\section{Conclusion}

In summary, the ideas presented in this paper will have influence on clinical proceedings like pressure measurements, interpretation of the results, quick diagnostic tests, just to name the most important ones. The implication of the findings should be extended in further research.

\section{Conflicts of interest}

The author declares that he has no conflict of interest.

\section{Acknowledgment}

The author thanks Patrycja Herbowssska-Tytro for help in the English translation and final preparation of the paper in terms of lexis and grammar.

\section{References}

1. Smoluchowski M (1906) Zur kinetischen theorie der brownschen molekularbewegung und der suspensionen. Annalen der Physik 21: 755-780.

2. Staroń W, Herbowski L, Gurgul H (2007) Optical analysis of suspended particles in cerebrospinal fluid (CSF) circulation. Proc. Of SPIE 6609.
3. Herbowski L, Gurgul H, Staron W (2009) Experimental determination of the Stern layer thickness at the interface of the human arachnoid membrane and the cerebrospinal fluid. Z Med. Phys 19: 189-192.

4. Herbowski L, Gurgul H (2011) The structure of the electric double layer of macromolecules suspended in human cerebrospinal fluid. J Neurol Neurophysiol 2: 108.

5. Staron W, Kubisz L, Herbowski (2012) Preliminary analysis of size distribution of objects suspended in normal cerebrospinal fluids in case of ventricular hydrocephalic enlargement and internal hydrocephalus. Acta Physica Polonica A 121: A-46-A-48.

6. Gurgul H, Herbowski L (2005) Preliminary determination of the influence of molecules concentration/shape and preesure on the viscosity fluid and the influence of posttraumatic subarachnoid bleeding on position - related changes of the cerebrospinal fluid pressure. Zeszyty Naukowe Uniwersytetu Szczecińskiego, Szczecin. Acta Physica 14: 21-38.

7. Herbowski L, Gurgul H (2008) Atmospheric pressure and basic parameters of intracranial volume-pressure homeostasis. Neurol Neurochir Pol 42: 332-337. [Crossref]

8. Herbowski L (2017) The major influence of the atmosphere on intracranial pressure: an observational study. Int J Biometeorol 61: 181-188.

9. Herbowski L, Gurgul H (2011) Thermodynamic approach to cerebrospinal fluid circulation. J Neurol Res 1: 215-218.

Copyright: $@ 2019$ Herbowski L. This is an open-access article distributed under the terms of the Creative Commons Attribution License, which permits unrestricted use, distribution, and reproduction in any medium, provided the original author and source are credited. 